(c) Evolutionary status of Pop. II Cepheids. Schwarzschild and Harm (Ap.J., 160, 341) calculated that type II Cepheids are in loops of final evolution off the asymptotic branch. This is supported by findings of Wallerstein (Ap.J. 160,345) from observations of Cepheids in many clusters, that these occur only in clusters with a strongly blue horizontal branch, but Kukarkin and Voroshilov (Astr. Cirk. Izdav. bjuro astr. Soob̌šc 617) disagree with some of these ideas from the standpoint of masses. Mengel (Coll. 21) also supports the earlier work for periods greater than 8 days, but finds a smaller number of loops. Kraft (Dudley Obs. Rept. 4, 69) suggested that Cepheids with shorter periods are evolving off the horizontal branch, also confirmed by Mengel.

\title{
12. Summaries
}

Excellent review papers in this field presented at the $I A U$ Colloquium $\mathbf{2 1}$ deserve special mention. M. W. Feast presented 'Observational Aspects of Slow Variables in Globular Clusters', L. Rosino 'Observational Aspects of RR Lyrae Variables in Globular Clusters', and P. Demarque 'Variable Stars and Evolution in Globular Clusters'.

\section{Variables in clusters of external galaxies}

As is to be expected from the great observational difficulties involved, the study of these variables proceeds slowly, and so far concerns only those of Magellanic Cloud clusters. The cluster NGC 1466, a red globular, lies between the two clouds at the distance of the Larger. Wesselink has found this to be very rich in variables $(M N R A S, 152,159)$. For the $43 \mathrm{RR}$ Lyrae stars he has published periods and light curves for 6. Norris (Coll. 21) has brought the number of periods to a total of 10 , of which 9 are $R_{a b}$ and $1 R$. He notes that the mean period of the $R R_{a b}$ 's is 0.533 , and that so far no clusters with longer ab type periods have been found in the Magellanic Clouds, suggesting that this system is not as evolved as the galaxy. The cluster NGC 1835, a compact cluster close to the LMC bar, has been found by Graham and Ruiz (Coll. 21) to be rich in variables. There are 21 definite, out of 49 suspected. Periods have been determined for 10,9 of which are $R_{\mathrm{ab}}$ and $1 \mathrm{RR}_{\mathrm{c}}$. The others are a long period, a possible Cepheid, and some more $\mathbf{R R}_{\mathbf{c}}$. Near the cluster NGC 1783 in which Gascoigne noted 3 variables in 1962, Graham (PAS Pacific, 82, 918) has found an eclipsing variable $2^{\prime}$ from the center, with period around 1 day or 2 days. Despite a careful search, he found no RR Lyrae variables on 60-inch Cerro Tololo plates. Gaposhkin (Payne Gaposchkin, Smithsonian Contrib. Astrophys. 13) has found two faint RR Lyrae stars, HV 13015 and 13016, in the faint cluster, HS 83 of the Large Cloud, and considers them members of the cluster. Demers (Coll. 21) with the University of Toronto 24-inch on Las Campanas has studied the colors of 6 Cepheids in 3 red globular clusters of the Large Cloud. These are the variables with periods over a day in and near NGC 1751, 1953 and 2121. Variables in these distant clusters constitute one of today's challenges to be met with the new equipment in the southern hemisphere.

\section{APPENDIX II}

\section{REPORT OF THE WORKING GROUP ON SUPERNOVAE}

By the end of 1969 the supernovae discovered since 1885 and included in Zwicky's master list had reached the number 260 . The rate of discovery at 12 participating observatories, in 1969 had reached about 15 supernovae per year. Since then, the same rate has been maintained as far as active supernovae are concerned, about 40 having been discovered between January 1, 1970 and May 13, 1972. In addition to these, however, 37 past supernovae were found on the original Palomar Sky Survey Plates and later comparison plates through a systematic search initiated by Zwicky a few years ago. The Master List, by May 13, 1972, consequently contained 336 bona fide supernovae. After further checks two more objects may have to be added to this list, that is a fifth supernova in NGC 6946, discovered by Rosino and one old one announced by Lovas as having been found on 
Field No. 155 (1955) of the Palomar Sky Survey prints. Among the 12 participating observatories, Asiago, Berne, Correlitos, Konkoly and Palomar have been most active in the search.

Among the 'live' supernovae, those whose spectra could be observed were all of the Types I and II. Among the objects found on the Palomar Sky Survey Plates, Zwicky's type V is also represented. Furthermore, two of the objects located on these plates were much bluer than any previously observed. It is therefore suspected that they belong to a new type VI or to the so far poorly known Zwicky Type IV.

Some difficulty has arisen as to the cause of the variability and the light eruption in compact galaxies such as have been observed for instance in I $\mathrm{Zw}$ 1. These may be due to the flare-up of supernovae, to collisions of stars in the system or to various other possible causes. I have proposed for the time being to keep separate lists and records for these objects but, in any case to announce their discovery and observed characteristics in the Bulletin for variable stars of Commission 27.

As to the frequency of supernovae it must be emphasized that the systematic searches conducted by the members of the working group of Commission 27 and by their associates mostly cover galaxies brighter than the indicative absolute magnitude $M_{p}=-15 \cdot 0$, and supernovae which at maximum become brighter than the indicative absolute magnitude $M_{p}=-140$. For these searches, the average frequency of one supernovae per galaxy per 350 years (Zwicky, Ap.J., 96, 28, 1942) still retains its validity, a conclusion that has recently been strikingly confirmed by the number of supernovae which have so far been located on the original Palomar 48-inch Schmidt Sky Survey Plates. The much higher frequencies derived recently by some authors include absolutely faint supernovae $\left(-11.0>M_{p}>-14.0\right)$ in a few nearby giant systems, that cannot be compared with the much lower frequencies derived for the brighter supernovae and from much more extensive and reliable data. These data also support the general conclusion that the frequency of occurrence is roughly proportional to the brightness of the parent galaxies and that, among these there are almost certainly some particularly fast producers of supernovae, the cause for this being at the present time a complete mystery (Zwicky, Supernovae, in Stars and Stellar Systems, Vol. VIII, University of Chicago Press, 1965).

It is interesting to note that of the five brightest supernovae found since 1885 four have occurred in small galaxies. Of the five only the supernova of 1885 in Messier 31 was fainter than the parent galaxy. It would also seem significant that two of the supernovae, which were all of them brighter than their parent galaxies appeared within 80 years in the irregular system NGC 5253.

The two most rewarding discoveries in the recent past have been the type II supernova discovered by Lovas in Messier 101 and the bright type I supernova found by Kowal in NGC 5253. Unfortunately the supernova in M 101 was imbedded in a bright emission line nebulosity. This has made the interpretation of the spectral features of the supernova in some respects somewhat uncertain. It will in particular be very important to disentangle the respective radio observations. Some investigators reported that the emission of radiowaves from the supernova was observed a short time after its outburst. If correct this would constitute an important new fact, since we had thought previously that the gas clouds emitted from a supernova of Type II would have to expand first for many years before a plasma would be formed tenuous enough to produce cyclotron radiation.

The supernova of Type I in NGC 5253 on the other hand was located far out from the main body of the parent galaxy. The many observations already made and still in progress of its light curve in various colours and the spectral investigations covering a large range from the infrared to the ultraviolet, as well as for the first time observations from a satellite should therefore yield much information of fundamental value. Among many other results, Professor J. B. Oke of the Palomar Observatory thinks indeed that with his scanner he has identified the permitted lines $\lambda 8542$, etc., and the forbidden lines $\lambda 7291$, etc., which are associated with the $\mathrm{H}$ and $\mathrm{K}$ lines of CaII.

These findings possibly confirm Zwicky's long standing conjecture that supernovae of the Type I are caused by the collapse of unique objects which symbolically may be called 'iron white dwarfs', that is, white dwarfs containing none of the light elements, such as hydrogen and helium. Implosions and symmetrical collapses of such dwarfs into neutron stars may be expected to produce similarly unique effects, such as small dispersion in the absolute magnitude of the resulting supernovae, 
distinctive light curves and spectra in which the lines of hydrogen and helium are absent and only lines of the heavier atoms such as calcium and iron show. If the observed velocity of expansion is really of the order of $13000 \mathrm{~km} \mathrm{sec}^{-1}$ as apparently derived by Oke, this also agrees qualitatively with that expected to be produced by the implosion of an iron white dwarf.

As to the original bodies producing the other four types of supernovae, no real progress seems to have been made yet, except that it appears fairly certain that supernovae of the Zwicky type III are not due to the collapse of any star but rather that of gas clouds of much greater mass.

Supernovae of the type I which have appeared in the Coma cluster (and other clusters of galaxies) show a remarkably small dispersion in apparent and therefore in absolute magnitudes. If this fact is confirmed by future observations, supernovae of the type I would therefore be the first and only cosmic objects available for the determination of large distances provided that we had a good value for their absolute brightness. Present indications for supernovae of the type I are that their photographic absolute magnitude at maximum is about $M_{p}=-18 \cdot 0$. The Lundmark-Hubble redshift constant consequently would be about $150 \mathrm{~km} \mathrm{sec}^{-1}$ million parsecs. In any event it should be emphasized that those who are deriving much smaller values through the use of sizes of HiI regions and luminosities of globular clusters and variable stars are deluding themselves, since the sizes and luminosities of all of these objects are not intrinsically fundamental and they are furthermore observationally unsuitable to gauge distances large enough for a reliable determination of the universal redshift constant.

Finally it should be mentioned that several new means have been developed to facilitate the search for supernovae. For instance, Wild at Zimmerwald now uses a twin Schmidt telescope which automatically will eliminate the difficulties caused by the interference of photographic defects. The large Tautenburg Schmidt telescope has now been equipped with a fullsize prismatic Schmidt plate which produces spectra of stars as faint as the apparent photographic magnitude $19 \cdot 0$. On the plates obtained supernovae may be recognized directly, eliminating thus the very laborious and timeconsuming (by blinking or other means) comparison of two different epoch plates. J. Strong and F. Zwicky had three $45 \mathrm{~cm} \times 45 \mathrm{~cm}$ transparent objective laminar gratings constructed for the same purpose. Finally Hynek at Correlitos and Colgate at Socorro have been constructing telescopes using television and photo-electronic scanning and computers. While Hynek's group has already discovered some supernovae, no news has been as yet received from Colgate's group at Socorro, N.M.

For the IAU meetings in Australia and Poland Professors Detre, Rosino and the Palomar group (Kowal, Oke, Sargent Zwicky) have some additional suggestions aiming at making the supernova search and analysis more effective than in the past.

F, ZWICKY

Chairman of the Working Group

\section{APPENDIX III}

REPORT ON THE SPECTRA OF VARIABLE STARS

\section{G. H. Herbig}

\section{General}

Götz and Wenzel $(M V S, 5,105 ; 6,35)$ have continued their work on the spectra of variable stars classified on objective-prism plates. The very extensive lists of peculiar, carbon, and emission-line stars contained in the Southern Objective-Prism Survey by Stock and Wroblewski (Pub. Dept. Astr., Univ. Chile, 2, No. 3) contain many objects that deserve detailed spectroscopic investigation. A list of 101 new northern M, C, and bright-line stars found at Haute Provence has been published by Barbier and Viale (AA Suppl, 2, 201).

\section{Red variables: Miras, semiregulars, $R V$ Tauris, and "infrared stars"}

The high level of new activity in this area is due to the discovery of infrared excesses and of 